\title{
Amplatzer Closure of Atrial Septal Defect and da Vinci Robot-Assisted Repair of Vascular Ring
}

\author{
R.G. Ohye, ${ }^{1}$ E.J. Devaney, ${ }^{1}$ J. Graziano, ${ }^{2}$ A. Ludomirsky ${ }^{2}$ \\ ${ }^{1}$ Division of Pediatric Cardiovascular Surgery, University of Michigan School of Medicine, Ann Arbor, MI 48109, USA \\ ${ }^{2}$ Division of Pediatric Cardiology, University of Michigan School of Medicine, Ann Arbor, MI 48109, USA
}

\begin{abstract}
Technology for minimally invasive approaches to congenital heart disease is a rapidly evolving field. This case report reviews a novel approach to combining two of the newer technologies available to treat a pediatric patient with an atrial septal defect (ASD) and a vascular ring. This report is the first to describe the use of the da Vinci surgical system to assist in a thoracoscopic procedure for a pediatric patient. The da Vinci assisted division of the vascular ring, joined with an Amplatzer closure of the ASD, demonstrates how maximum benefit can be obtained for patients by combining emerging technologies.
\end{abstract}

Keywords: Atrial septal defect — Vascular ring Amplatzer — da Vinci

\section{Case Report}

A 6-year-old male presented to the C. S. Mott Children's Hospital at the University of Michigan with a diagnosis of a secundum-type atrial septal defect (ASD) and a vascular ring consisting of a right aortic arch with left ligamentum arteriosus. He was recently diagnosed after work up for a murmur noted on physical exam. His mother reported that he occasionally needed to stop strenuous activity for "shortness of breath," thought to be due to reactive airway disease. He denied any stridor, wheezing, or dysphagia. His previous medical history was significant only for the diagnosis of reactive airway disease. He was on no scheduled medications.

Physical exam revealed a healthy-appearing young male in no distress. Pertinent findings included clear and equal breath sounds bilaterally. There was a normal S1 and fixed, split S2 with a grade II/VI systolic ejection murmur at the left upper sternal border. Chest radiograph showed mild cardiomegaly with slightly

Correspondence to: R.G. Ohye, F7830 Mott/0223, 1500 East Medical Center Drive, Ann Arbor, MI 48109, USA, email: ohye@umich.edu increased pulmonary vascular markings and a right aortic arch. Doppler/echocardiogram demonstrated a $6.5-\mathrm{mm}$ secundum-type ASD and a right aortic arch with aberrant left subclavian artery. Computed tomography of the chest confirmed the vascular ring consisting of a right aortic arch with an aberrant left subclavian artery arising from a Kommerell's diverticulum (Fig. 1).

The patient was admitted to the pediatric cardiology service, at which time he under went closure of his ASD with an Amplatzer septal occluder (AGA Medical, Golden Valley, MN, USA) (Fig. 2). Postprocedure transesophageal Doppler/echocardiography revealed no significant residual shunting (Fig. 3). The following day, the patient was taken to the operating room and placed in a right lateral decubitus position. A bronchial blocker was employed for single right lung ventilation to assist in exposure. The division of the left ligamentum arteriosus was performed using the da Vinci surgical system (Intuitive Surgical, Sunnyvale, CA, USA) via four ports (Fig. 4). The ports included a $12-\mathrm{mm}$ port for the camera, a $10-\mathrm{mm}$ port for a lung retractor, and 2 to $8-\mathrm{mm}$ ports for the operating instruments. The ligamentum arteriosus was circumferentially mobilized, clipped with an endoscopic clip applier in case of residual patency, and divided. Fibrous bands around the esophagus and tracheal were divided, and a small Kommerell's diverticulum was mobilized. The patient had an unremarkable post operative course and was discharged on postoperative day 2 .

\section{Discussion}

Technologies for minimally invasive therapy are a rapidly growing segment of the field of congenital heart disease. A simple MEDLINE search for minimally invasive cardiac or thoracic surgery manuscripts reveals more than 1000 citations for 20012002 alone. The goals of these techniques are to safely decrease morbidity, cost, patient discomfort, and length of convalescence with improved patient satisfaction and cosmetic result. Two of the newer techniques available are the use of the Amplatzer septal occluder for catheter-based closure of (ASDs) and the da Vinci surgical system, which is an operative robot to assist in minimally invasive procedures.

The Amplatzer has been used with excellent success for the closure of ASD. Dong and associates 

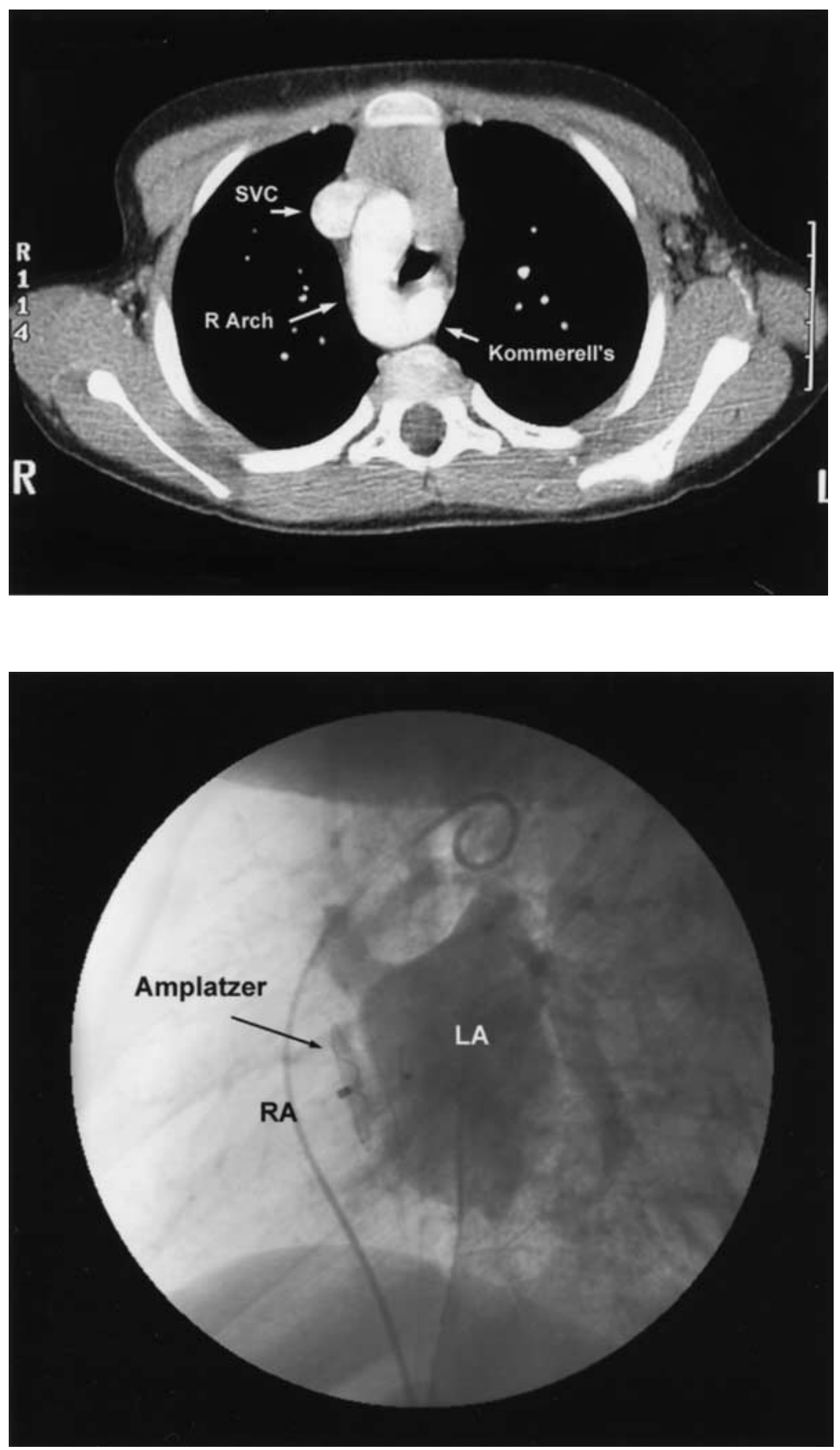

Fig. 1. Axial image of the chest computed tomography demonstrating the right aortic arch and origin of the aberrant left subclavian from a Kommerell's diverticulum. $S V C$, superior vena cava.
Fig. 2. Lateral view of the levophase of a pulmonary artery injection showing the Amplatzer to be in good position with no residual shunting. $L A$, left atrium; $R A$, right atrium.
[1], in a multinstitutional study comparing transcatheter and surgical closure of ASD, found a $95.7 \%$ success rate for device closure compared to $100 \%$ for surgery $(p=0.006)$. The rate of major complication, not including failure of closure, was $1.6 \%$ (7/442) for device closure and $5.4 \%(8 / 154)$ for the surgical group $(p=0.03)$. Mean length of stay was $1.0 \pm 0.3$ days for the device group and $3.4 \pm 1.2$ days for the surgery group $(p<0.001)$. A similar decrease in length of stay was demonstrated by Hughes and colleagues [2] in a prospective study comparing 43 children who underwent Amplatzer device closure and 19 who underwent surgery. They found median hospital stays to be 88 hours (range, 78-112) compared to 29 hours (range, 28-30) for the surgical and device groups, respectively $(p<0.01)$. Although the 

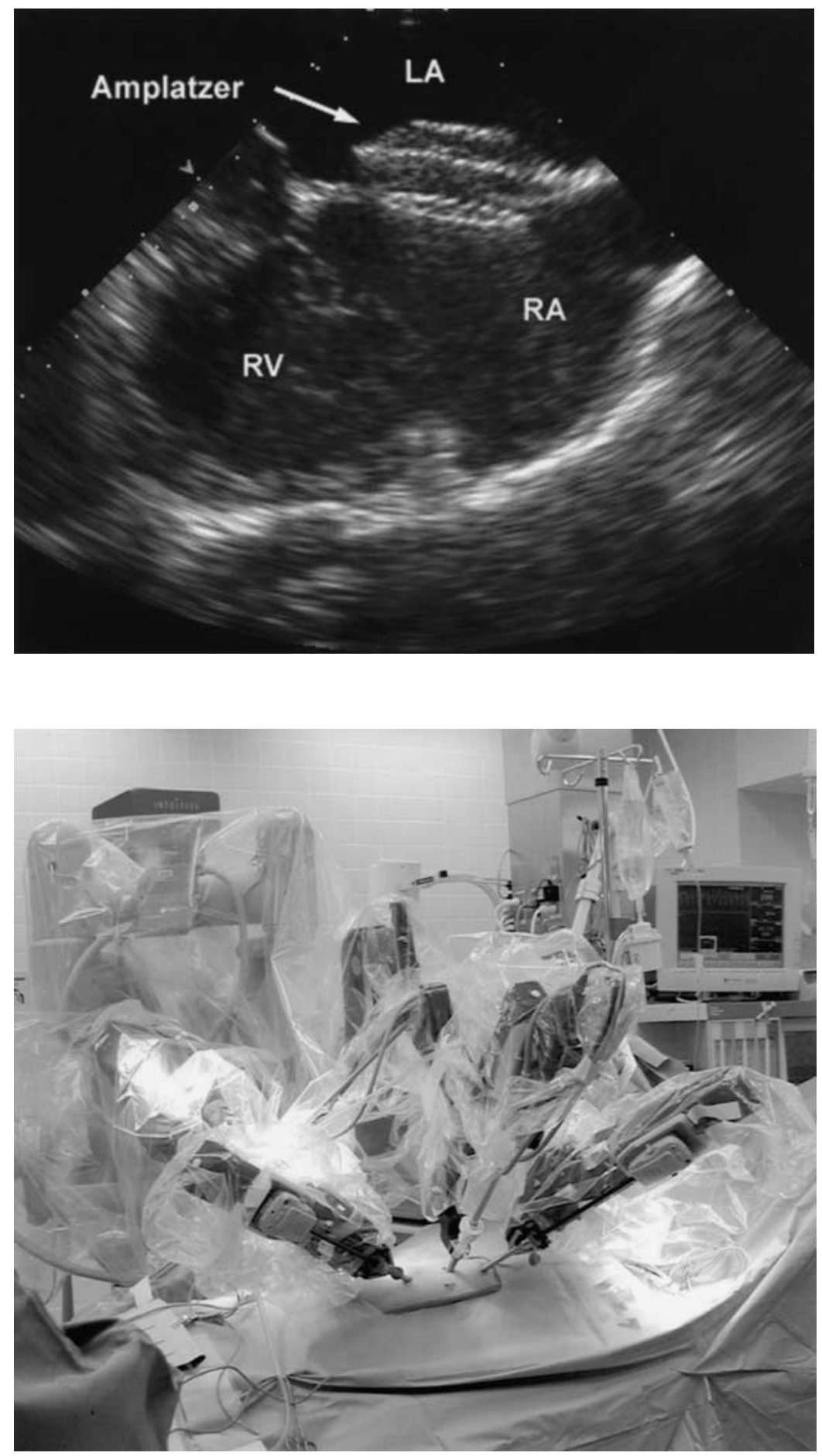

Fig. 3. Transesophageal echocardiogram showing the Amplatzer to be in good position. $L A$, left atrium; $R A$, right atrium; $R V$, right ventricle.
Fig. 4. Intraoperative photograph showing the da Vinci Surgical System in place with the two instrument arms (left and right) and camera (center arm) entering the left chest with the patient in a right lateral decubitus position. median cost of the procedures was similar, total hospital costs were slightly higher for the surgical group at Aus \$12,969 (range, \$11,569-\$14,215) compared to Aus $\$ 11,845$ (range, $\$ 10,669-\$ 12,555$ ) $(p=0.03)$.

In adults, the use of the da Vinci system has been reported for several different applications, including lobectomy, resection or oversewing of bullae, lung and mediastinal tumor removal, and ASD closure [3-5]. This report is the first to describe the use of the da Vinci system to perform a pediatric cardiothoracic procedure, a division of a vascular ring. By combining this thoracoscopic procedure with a catheter-based closure of the 
ASD, the need for open-heart surgery and cardiopulmonary bypass was avoided.

\section{References}

1. Dong Z-D, Hijazi ZM, Kleinman CS, Silverman NH, Larntz K (2002) Comparison between transcatheter and surgical closure of secundum atrial septal defect in children and adults. Results of a multicenter nonrandomized trial. $J$ Am Coll Cardiol 39:1836-1844

2. Hughes ML, Maskell G, Goh TH, Wilkinson JL (2002) Prospective comparison of costs and short term health outcomes of surgical versus device closure of atrial septal defect in children. Heart 88:67-70

3. Melfi FM, Menconi GF, Mariani AM, Angeletti CA (2002) Early experience with robotic technology for thoracoscopic surgery. Eur J Cardiothorac Surg 21:864-868

4. Yoshino I, Hashizume M, Shimada M, Tomikawa M, Sugimachi K (2002) Video-assisted thoracoscopic extirpation of a posterior mediastinal mass using the da Vinci computer enhanced surgical system. Ann Thorac Surg 74:1235-123

5. Argenziano, M, Oz, MC, Kohmoto, T, Morgan, J, Dimitui, J, Mongero, L, Beck, J, Smith, CR (2003) "Totally endoscopic atrial septal defect repair with robotic assistance" CIRC 108(Suppl 1): II 191-194

\section{Around PediHeart: Management of Ventricular Septal Defects}

In May, Doctor Juan Villafane sent out a questionnaire to PediHeart members regarding ventricular septal defect (VSD) management in children. The following is a summary of responses, with some editorial additions.

CLASSIFICATION by size relative to the aortic diameter was advocated: small $<40 \%$; moderate $40-60 \%$; large $>60 \%$ of the aorta. Other classifications used include restrictive VSDs where the RV pressure is $<L V$ pressure, or non-restrictive when the pressures are equal.

MEDICAL MANAGEMENT: therapy may not be required in the majority of VSDs, since $>1 / 2$ will close spontaneously in the first year [1]. For those in congestive failure, diuretics and ACE-inhibitors were agreed upon by the responders, and about half of us would use digoxin —or even start with it. Half of us would not use betablockers. Some would use hypercaloric formula, with or without a nasogastric tube, for failure to gain.

Trans-catheter closure is now feasible but published figures suggest somewhat increased mortality compared to surgical closure.

LV function is usually not impaired with a VSD and, beyond shortening-fraction by echocardiography, it is rarely necessary to define. However, in VSD patients with failure and respiratory distress, Dr. Villafane recommended monitoring these patients for levels of brain natiuretic peptide.

SURGICAL MANAGEMENT: Indications for surgical closure include pulmonary hypertension, uncontrolled failure under vigorous medical management, and subpulmonary VSD with aortic prolapse. Although some advocate closure based on failure to thrive alone, there was no documentation to support this. Pulmonary banding is still being used for complex VSDs such as Swiss-cheese types. Children with Down syndrome and a VSD (with or without endocardial cushion defect) are at increased risk of pulmonary hypertension and therefore require careful follow-up in the first 6 months, and may require early intervention.

Consensus on indications for elective VSD closure was lacking. At one extreme, some routinely operate when the infant attains $4 \mathrm{~kg}$, others simply at 6 to 48 months of age in the presence of a shunt when QP/QS is $>2$. Some of us are patient as long as the child has no pulmonary hypertension or congestive failure, having documented spontaneous closure in asymptomatic 20-year-olds.

Warren Guntheroth, M.D.

\section{Reference}

1. Moe DG, Guntheroth WG (1987) Spontaneous closure of uncomplicated ventricular septal defect. Am J Cardiol 60:674-678 